\title{
Reduction of operating voltage in organic light-emitting diode by corrugated photonic crystal structure
}

\section{$\operatorname{AUTHOR}(S)$ :}

Fujita, M; Ueno, T; Ishihara, K; Asano, T; Noda, S; Ohata, H; Tsuji, T; Nakada, H; Shimoji, N

\section{CITATION:}

Fujita, M ... [et al]. Reduction of operating voltage in organic light-emitting diode by corrugated photonic crystal structure. APPLIED PHYSICS LETTERS 2004, 85(23): 5769-5771

\section{ISSUE DATE:}

2004-12-06

URL:

http://hdl.handle.net/2433/50208

\section{RIGHT:}

Copyright 2004 American Institute of Physics. This article may be downloaded for personal use only. Any other use requires prior permission of the author and the American Institute of Physics. 


\title{
Reduction of operating voltage in organic light-emitting diode by corrugated photonic crystal structure
}

\author{
Masayuki Fujita, ${ }^{a}$ Tetsuya Ueno, Kuniaki Ishihara, Takashi Asano, and Susumu Nodab) \\ Department of Electronic Science and Engineering, Kyoto University, Kyotodaigaku-katsura, \\ Nishikyo-ku, Kyoto 615-8510, Japan \\ Hiroshi Ohata, Taishi Tsuji, and Hitoshi Nakada \\ Pioneer Corporation, Corporate Research and Development Laboratories, 1-1, Fujimi 6 Chome, \\ Tsurugashima, Saitama 350-2288, Japan \\ Noriyuki Shimoji \\ Rohm Corporation Limited, New Material Devices Research and Development Center, 21, \\ Saiin Mizosaki-cho, Ukyo-ku, Kyoto 615-8585, Japan
}

(Received 17 May 2004; accepted 21 October 2004)

\begin{abstract}
A reduction of the operating voltage is achieved for an organic light-emitting diode containing a corrugated photonic crystal structure fabricated by the etching of an indium-tin-oxide anode layer. This is due to a partial reduction in the thickness of the organic layer. The light extraction efficiency can be also improved due to the diffraction of confined light by the photonic crystal effect. The voltage reduction is demonstrated in combination with an improvement in the luminance efficiency at constant current for the fabricated device. (C) 2004 American Institute of Physics.
\end{abstract}

[DOI: $10.1063 / 1.1836867]$

Organic light-emitting diodes (OLEDs) are very promising devices for use in flat panel displays and illumination applications due to the possibility of fabricating very thin flexible structures that emit light over large areas with high brightness and low power consumption. ${ }^{1,2}$ The realization of highly efficient devices is one of the most critical issues for such applications. The luminance power efficiency ${ }^{3}$ (related to the internal quantum efficiency, the light extraction efficiency, and the electrical characteristics) is important for practical purposes (in particular mobile). Thus far, the internal quantum efficiency, an intrinsic property of the organic material, has been improved by the use of phosphorescent harvesters. ${ }^{4}$ The light extraction efficiency is limited to $\sim 20 \%$ due to total internal reflection. ${ }^{5-8}$ From theoretical calculations for typical OLED structures, $\sim 50 \%$ of the light is guided and trapped in the high refractive index indium-tinoxide (ITO) anode and organic layers. In order to improve the light extraction efficiency, it is therefore important to find a method for extraction of light confined to such guided modes. One promising approach is the incorporation of a photonic crystal (PC), a periodic dielectric structure inside which light can be controlled, into the OLED.

This method has recently resulted in improvements to the luminance power efficiency in an OLED. ${ }^{9}$ The OLED structure itself, including the ITO and organic and metallic cathode layers, is periodically corrugated to form a PC. In this way, diffraction of confined light can be maximized and is expected to be stronger than in previous reports. ${ }^{10}$ In this organic device, electroluminescence was obtained without serious problems, contrary to the expected results for a structure that is not flat. However, the effect of the corrugated structure on the electronic characteristics has not been investigated until now. In this letter, we discuss the electronic characteristics of the device and show that the current-

\footnotetext{
${ }^{a}$ Electronic mail: fujita@qoe.kuee.kyoto-u.ac.jp

${ }^{b)}$ Electronic mail: snoda@kuee.kyoto-u.ac.jp
}

voltage characteristics can be improved together with the light extraction efficiency.

Samples were fabricated using the same procedure in Ref. 9; electron-beam lithography to write two-dimensional periodic square lattice pattern, plasma etching of a part of $150 \mathrm{~nm}$ thick ITO layer, evaporation of $130 \mathrm{~nm}$ thick organic layer in a vacuum chamber, and packing with a desiccant in a nitrogen atmosphere. The fabricated emission area was $2 \mathrm{~mm} \times 2 \mathrm{~mm}$. The period of the square lattice $(a)$ was varied in the range of 300-1000 $\mathrm{nm}$ and the mean diameter of the etched holes was set to be 100-300 nm. Reference samples having conventional OLED structures (without patterned ITO) were also fabricated on the same substrate. Figure 1 shows cross-sectional views of the fabricated sample.

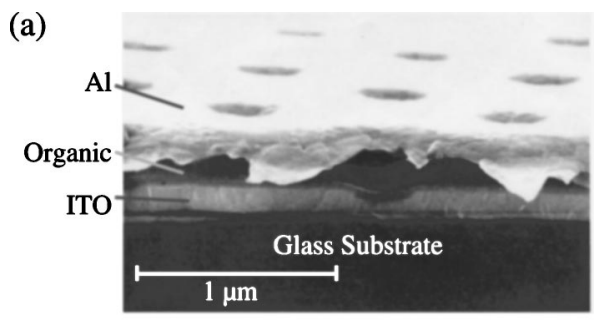

(b)

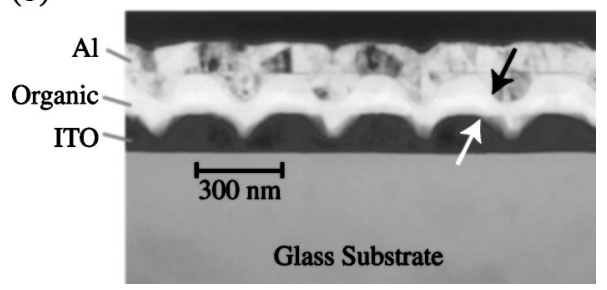

FIG. 1. Cross-sectional views of the samples fabricated with a corrugated PC micropattern. (a) Bird's-eye view using a scanning electron microscope. The apparent damage at the edge of the metal layer resulting from the cleaving process. (b) Side view using a transmission electron microscope. The interval between arrows indicates the points of minimum thickness in the organic layer. 
(a)

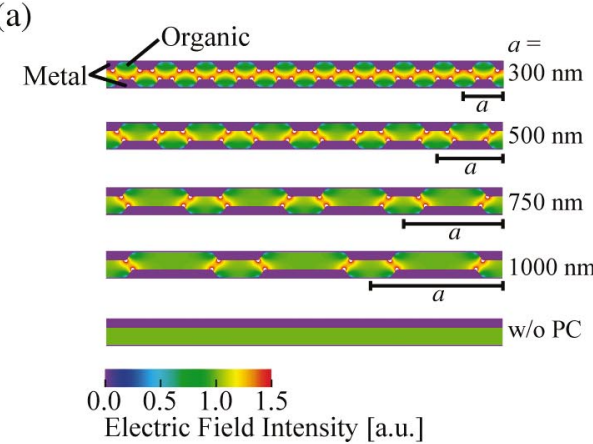

(b)

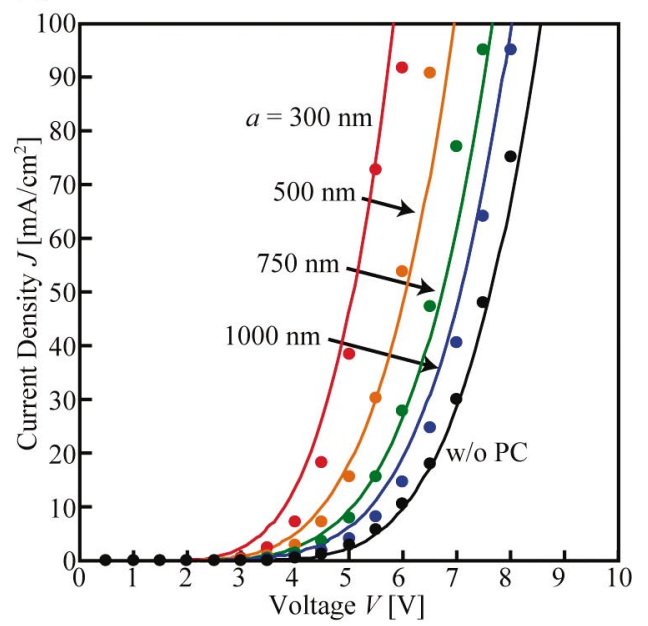

FIG. 2. (Color) Electric characteristics for various PC period $a$. Period $a$ is varied between 300 and $1000 \mathrm{~nm}$. The etch depth of ITO is fixed at $60 \mathrm{~nm}$. Corresponding minimum thickness of organic layer is $\sim 90 \mathrm{~nm}$. (a) Simulated static electric-field intensity distribution. The cross section was modeled on fabricated samples. The applied voltage is constant and the electricfield intensity is normalized by that of conventional OLED. (b) $J-V$ for different samples. Plots and lines denote results for experiement and calculation, respectively.

The sidewall of the etched ITO was tilted at an angle of $\sim 60^{\circ}$ to the substrate plane. Therefore, the organic and $\mathrm{Al}$ metallic-cathode layers were evaporated onto the sidewall of the etched ITO and no discontinuity in the organic layer was observed. The thickness of the organic layer (the distance between the anode and cathode) $t$ varies periodically due to the sample corrugation. To investigate the effect of this modified structure on the electric characteristic, we simulated the static electric-field distribution $\mathbf{F}$ of the cross section of the PC-OLED by solving the Laplace equation of $\nabla^{2} \phi=0$, and $\mathbf{F}=-\nabla \phi$, where $\phi$ is an electrostatic potential. Here, we assumed no electric field in metal electrodes and periodicity of the PC as boundary conditions. As shown in Fig. 2(a), the electric field intensity $|\mathbf{F}|$, which is almost inversely proportional to the distance between the electrodes, is enhanced at the minimum thickness region of the organic layer in the PC structure. Figure 2(a) also indicates that this enhancement per unit area becomes more remarkable as the PC period $a$ becomes smaller.

The fabricated samples were measured at room temperature using a combination of dc power supply, digital multimeter, and a TOPCON SR-1 spectroradiometer. Figure 2(b) shows the current density-voltage $(J-V)$ characteristics for samples with different PC periods. Figure 2(b) indicates that the voltage required to maintain a constant current decreases as the PC period becomes smaller. In comparison with conDownloaded 04 Mar 2008 to 130.54.110.22. Redistribution subject (a)

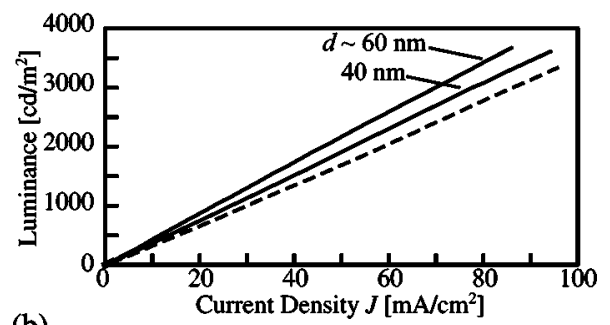

(b)

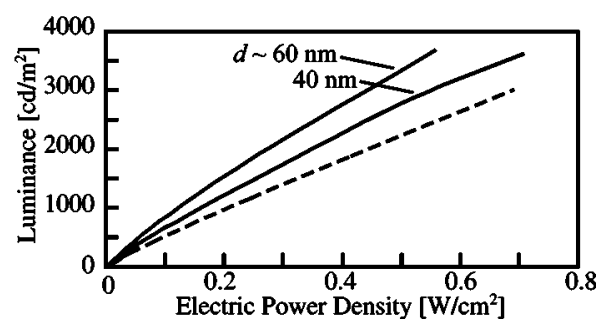

FIG. 3. Luminance characteristics for different samples. Solid and dashed lines correspond to results for the $\mathrm{PC}$ and conventional samples, respectively. The ITO etch depth $d$ is varied. Corresponding minimum thickness of organic layer for $d \sim 40 \mathrm{~nm}$ and $d \sim 60 \mathrm{~nm}$ are $\sim 100 \mathrm{~nm}$ and $\sim 90 \mathrm{~nm}$, respectively. The period $a$ is fixed at $300 \mathrm{~nm}$. (a) Luminance vs current density. (b) Luminance vs supplied electric power density.

ventional OLED structures, the operating voltage (e.g., for $J=50 \mathrm{~mA} / \mathrm{cm}^{2}$ ) is reduced by $30 \%$ for a PC-OLED with $a$ $=300 \mathrm{~nm}$. Alternatively, the current density at constant voltage (e.g., for $V=5 \mathrm{~V}$ ) is approximately ten times higher than in conventional structures. One may intuitively think that this effect is simply due to the increase in surface area of the electerode. However, this cannot explain the phenomena observed in Fig. 2 since the surface area is only $\sim 1.4$ times that of a conventional structure. Here, the current density $J$ of the OLED was expressed with the Fowler-Nordheim tunneling injection model $^{11,12}$ as:

$$
J=k_{1}|\mathbf{F}|^{2} \exp \left(-k_{2} /|\mathbf{F}|\right),
$$

where $k_{1}$ and $k_{2}$ are constants related to the material property. From $J-V$ characteristics of fabricated conventional OLED sample (the electric-field intensity is calculated as $|\mathbf{F}|=V / t$ ), we obtained $k_{1}=1.0 \times 10^{-11}\left[\mathrm{~A} / \mathrm{V}^{2}\right]$ and $k_{2}=2.5$ $\times 10^{8}[\mathrm{~m} / \mathrm{V}]$ as fitting parameters. Equation (1) indicates that a nonlinear decrease in the operating voltage $V$ is expected when the thickness $t$ is reduced at constant current. The $J-V$ characteristics of PC samples can be estimated by using Eq. (1) taking into account of the simulated electric-field intensity in Fig. 2(a). The calculation results are well coincident with the experimental ones, as shown in Fig. 2(b). Thus, we can conclude that electric characteristics of PC-OLEDs become more improved by the enhancement of electric-field intensity as the PC period becomes smaller due to the partial reduction of organic layer thickness.

Figure 3(a) shows an example of the luminance characteristic as a function of current density. The period of $300 \mathrm{~nm}$ in the PC samples corresponds to the calculated modal wavelength of the guided mode, which is determined by the wavelength of light emitted from the $\mathrm{Alq}_{3}$ [tris- (8-hydroxyquinoline) aluminum] layer, the refractive index, and the thickness of the OLED structure. The luminance of the PC samples is improved in comparison with the conventional structures. When the PC period is equal to the wavelength of the guided modes in the medium, waves propagating along o AlP license or copyright; see http://apl.aip.org/apl/copyright.jsp 


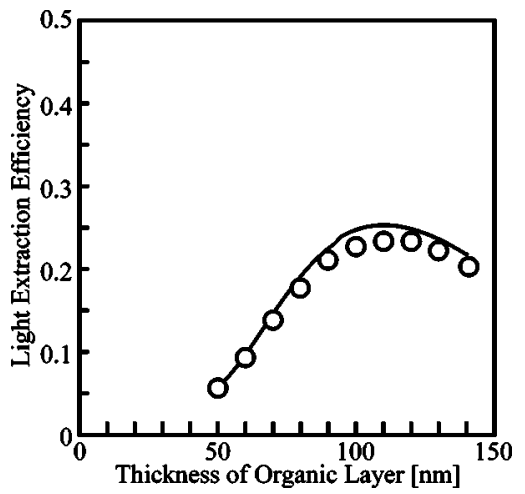

FIG. 4. Light extraction efficiency for conventional OLED as a function of the thickness of the organic layer, calculated by the finite-difference timedomain method (circles) and the mode expansion method (line). The structure consists of a light-emitting/electron transport layer (EML/ETL), a hole transport layer (HTL), an ITO anode, and a glass substrate. The refractive indices of the EML/ETL, HTL, ITO, and glass are assumed to be 1.70, 1.67, 2.0 , and 1.5 , respectively, at wavelength $524 \mathrm{~nm}$ which corresponds to the central emission wavelength of the EML. The thickness of the HTL and the ITO are 40 and $150 \mathrm{~nm}$, respectively. (The detailed method of calculation is given in Refs. 7 and 8.)

the in-plane direction of the sample are emitted normally to the surface of the device, since the Bragg diffraction condition is satisfied. ${ }^{13}$ Because the total internal reflection condition at the device surface (the glass substrate-air interface) for diffracted waves is broken, the diffracted wave is no longer confined inside the glass substrate. Thus, an improvement in the light extraction efficiency should be expected. The luminance efficiency for the PC sample is further improved by increasing the ITO etch depth $d$, due to the enhancement of the optical confinement factor in the PC layer. An increase in efficiency by a factor of $\sim 1.2$ is observed for the sample with $d \sim 60 \mathrm{~nm}$. In total, the luminous power efficiency is improved by a factor of 1.5 compared to that of conventional structures, as shown in Fig. 3(b). Here, Eq. (1) suggests that, even for conventional structures, the operating voltage can be continually reduced by decreasing the thickness $t$, until problems with short circuiting occur. However, the light extraction efficiency will be degraded by the reduction in thickness of conventional OLED structures, due to optical interference effects ${ }^{14,15}$ related to the distance between the dipole and the metallic cathode, as shown in Fig. 4. In contrast, the PC-OLED structures in this study can reduce the operating voltage while improving the light extraction efficiency, as discussed above. In addition, when a microstructure, whether it is periodic or not, is smaller than the wavelength of emitted light, the effective thickness of the organic layer can be approxiemated by its mean thickness. There can be an effective thickness which optimizes the light extraction efficiency. While the operating voltage at constant current is determined and minimized in the thinner region of the organic layer, as discussed above.

In summary, we have demonstrated not only low voltage operation but also high light extraction efficiency in OLEDs possessing a corrugated PC structure etched on the ITO layer. A 30\% reduction of the voltage at constant current compared to conventional structures has been achieved due to the partial reduction of the organic layer thickness. In addition, the luminance efficiency with respect to the current was also enhanced due to the PC light extraction effect. Consequently, the luminous power efficiency has also been improved. Thus, these OLED devices combine high brightness with low power consumption. We believe that further optimization of the structure should be possible, resulting in even greater efficiency.

The authors would like to thank Dr. A. Chutinan and Professor A. Toshimitsu of Kyoto University, K. Handa of Mitsubishi Chemical Corp., and Dr. K. Chikuma of Pioneer Corp., for helpful suggestions and encouragement. This work was supported by the Strategic University/Industry Alliances of International Innovation Center of Kyoto University and also, in part, by a grant-in-aid for scientific research (Grant No. 15GS0209) from the Ministry of Education, Science, and Culture, Japan. One of the authors (M.F.) was supported by a Research Fellowship of the Japan Society for Proemotion of Science (No. 15004417).

${ }^{1}$ C. W. Tang and S. A. VanSlyke, Appl. Phys. Lett. 51, 913 (1987).

${ }^{2}$ J. H. Burroughes, D. D. Bradley, A. R. Brown, R. N. Marks, K. Mackay, R. H. Friend, P. L. Burns, and A. B. Holmes, Nature (London) 347, 539 (1990).

${ }^{3}$ S. R. Forrest, D. D. C. Bradey, and M. E. Thompson, Adv. Mater. (Weinheim, Ger.) 15, 1043 (2003).

${ }^{4}$ M. A. Baldo, D. F. O’Brien, Y. You, A. Shoustikov, S. Sibley, M. E. Thompson, and S. R. Forrest, Nature (London) 395, 151 (1998).

${ }^{5}$ G. Gu, D. Z. Garbuzov, P. E. Burrows, S. Venkatesh, S. R. Forrest, and M. E. Thompson, Opt. Lett. 22, 396 (1997).

${ }^{6}$ J. S. Kim, P. K. H. Ho, N. C. Greenham, and R. H. Friend, J. Appl. Phys. 88, 1073 (2000).

${ }^{7}$ A. Chutinan, M. Fujita, W. Kunishi, T. Ueno, K. Ishihara, T. Asano, and S. Noda, Ext. Abstr. Jpn. Soc. Appl. Phys. Relat. Soc. 50, 1408 (2003).

${ }^{8}$ A. Chutinan, K. Ishihara, T. Asano, M. Fujita, and S. Noda (unpublished).

${ }^{9}$ M. Fujita, T. Ueno, T. Asano, S. Noda, H. Ohata, T. Tsuji, H. Nakada, and N. Shimoji, Electron. Lett. 39, 1750 (2003).

${ }^{10}$ Y. J. Lee, S. H. Kim, J. Huh, G. H. Kim, Y. H. Lee, S. H. Cho, Y. C. Kim, and Y. R. Do, Appl. Phys. Lett. 82, 3779 (2003).

${ }^{11}$ R. H. Fowler and L. Nordheim, Proc. R. Soc. London, Ser. A 119, 173 (1928).

${ }^{12}$ T. Oyamada, C. Maeda, H. Sasabe, and C. Adachi, Jpn. J. Appl. Phys., Part 2 42, L1535 (2003).

${ }^{13}$ S. Noda, Y. Yokoyama, M. Imada, A. Chutinan, and M. Mochizuki, Science 293, 1123 (2001).

${ }^{14}$ T. Tsutsui, C. Adachi, S. Saito, M. Watanabe, and M. Koishi, Chem. Phys. Lett. 182, 143 (1991).

${ }^{15}$ M. Mastumura and T. Furukawa, Jpn. J. Appl. Phys., Part 1 41, 2742 (2002). 